\title{
TEST YOURSELF: Answer psoriatic onycho-pachydermo periostitis (POPP)
}

\author{
Sarath Bethapudi • Jill Halstead • Zoe Ash • \\ Dennis McGonagle • Andrew J. Grainger
}

Published online: 5 July 2013

(C) ISS 2013

Painless swollen great toe.

\section{Diagnosis}

Psoriatic onycho-pachydermo periostitis (POPP)

\section{Discussion}

Psoriatic arthritis is a form of seronegative arthropathy that usually occurs in association with cutaneous manifestations, including skin psoriasis, nail changes or both.

The case presentation can be found at doi: 10.1007/s00256-013-1681-9

S. Bethapudi · A. J. Grainger

Department of MSK Radiology, Leeds Teaching Hospitals,

Leeds, West Yorkshire, UK

J. Halstead · Z. Ash · D. McGonagle · A. J. Grainger

NIHR Leeds Musculoskeletal Biomedical Research Unit,

Leeds Teaching Hospitals, Leeds, West Yorkshire, UK

J. Halstead · Z. Ash • D. McGonagle

Leeds Institute of Rheumatic and Musculoskeletal Medicine,

University of Leeds, Leeds, West Yorkshire, UK

\section{S. Bethapudi $(\bowtie)$}

Clinical Fellow in Musculoskeletal Radiology, Leeds Teaching Hospitals NHS Trust, Chapel Allerton Hospital, Chapeltown Road, Leeds LS7 4SA, West Yorkshire, UK

e-mail: bethapudi@gmail.com

A number of common subtypes of psoriatic arthritis are recognized, including mono or oligoarthritis, symmetric polyarthritis and spondyloarthritis. The rarer forms include SAPHO syndrome and POPP [1-3]. POPP is primarily a clinical diagnosis and is characterized clinically by psoriatic onychodystrophy, with marked periarticular soft tissue thickening (Fig. 1).

The original case series of four patients with POPP, described by Fournie B in 1989, manifested in the great toe and hence the condition was then referred to as POPP of the great toe [4]. Since then, POPP has been reported in various small joints of the digits in both upper and lower extremities [5], although the majority manifest in the great toe.

Radiographic findings in POPP include intra- and periarticular erosions, new bone formation including periostitis, bony protuberances, soft tissue prominence (dactylitis or sausage digits), calcification at ligament and tendinous insertions in keeping with enthesopathy $[1,6,7]$ (Fig. 2)

Given that POPP commonly involves the great toe of the foot, it is occasionally difficult to exclude infection on clinical grounds alone. MRI in such cases can provide additional useful information, which may be vital in differentiating the two conditions. Contrast is not routinely required, but may be administered in cases where active synovitis or osteomyelitis is clinically suspected.

MRI findings in POPP can be grouped into bone and soft tissue changes. Bony changes include periostitis and osteitis. On imaging, periostitis is seen as low signal periosteal thickening on T1-W imaging, while osteitis is seen as bone marrow edema, which manifests as intraosseous intermediate to low 


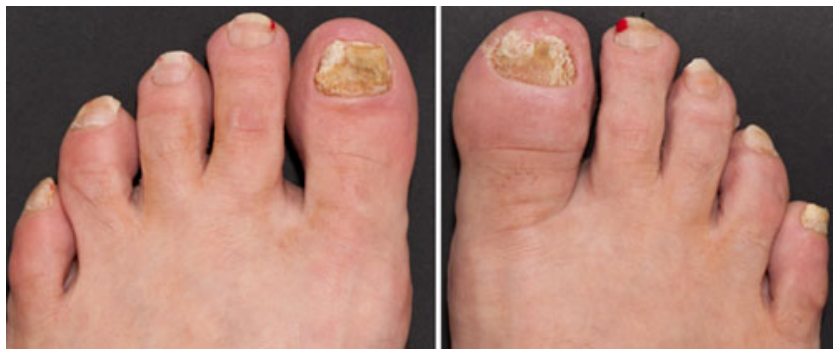

Fig. 1 Photographs of right and left toes show dystrophic nail changes in both the left and right great toes related to background history of psoriasis. However, the symptomatic right great toe is swollen and edematous in keeping with active dactylitis

signal on T1-W imaging and high signal on T2-W and fluidsensitive sequences. The bone marrow edema is usually confined to the distal phalanx of the affected digit and there is no joint space involvement [7] (Figs. 3, 4). The soft tissue swelling demonstrates intermediate to low signal on both T1-W and $\mathrm{T} 2-\mathrm{W}$ and fluid-sensitive sequences with no associated edema. The intermediate to low signal intensity demonstrated by the

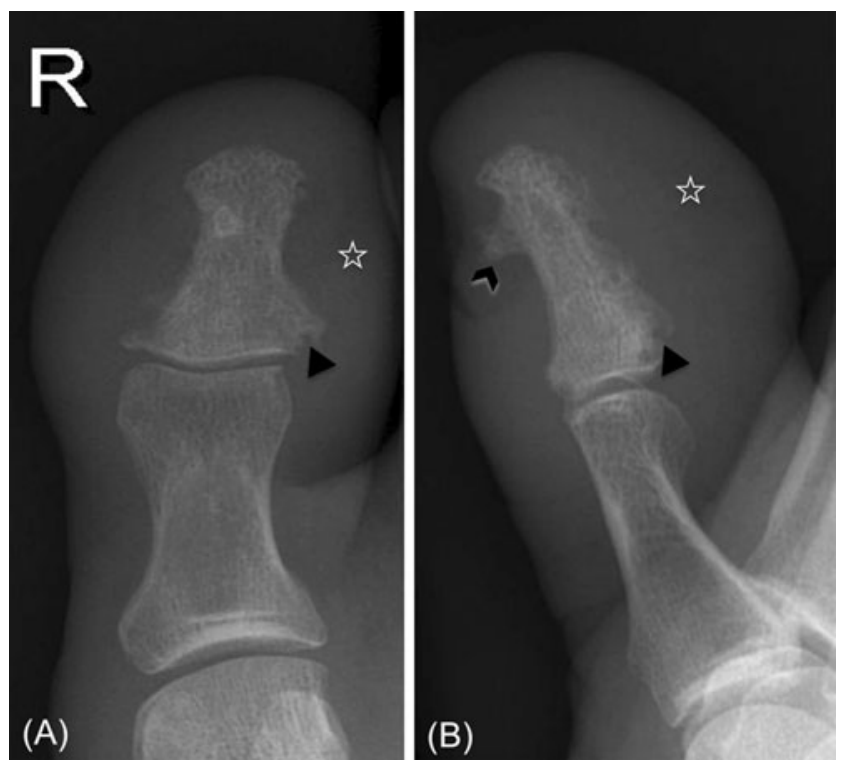

Fig. 2 Frontal (a) and lateral (b) radiographs of a 55-year-old female patient presenting with painless, swollen great toe demonstrate intraarticular erosions at the distal inter-phalangeal joint (black solid triangles) with sub-ungual bony exostosis (solid black chevron) and prominent periarticular soft tissues (hollow white star)
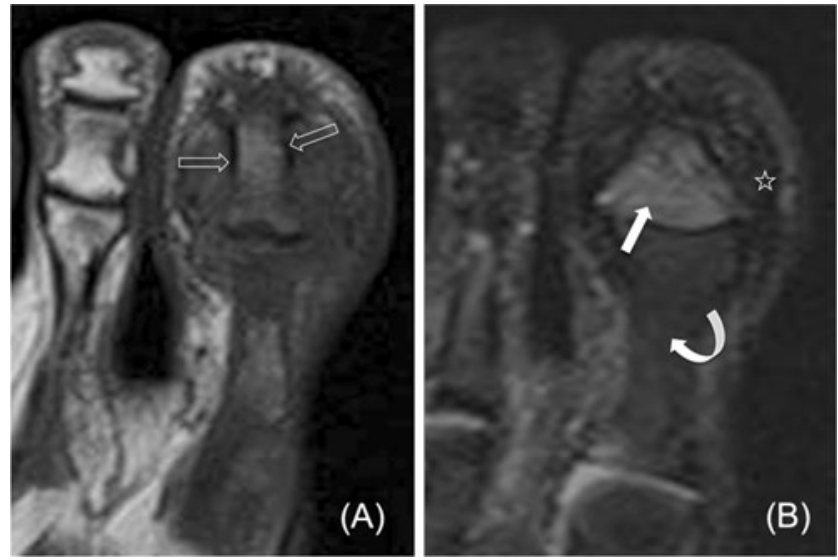

Fig. 3 MRI coronal T1W (a) and coronal T2-W fat-suppressed (b) demonstrate prominent periosteal thickening (hollow white arrows), bone marrow oedema (solid white arrow) and non-oedematous paraossesous soft tissue thickening (star) confined to the distal phalanx of great toe. Note the normal appearances of the proximal phalanx with normal marrow signal (curved white arrow). In contrast to osteomyelitis, abnormality in POPP does not cross the joint

prominent thickened periarticular and paraosseous soft tissues can be explained histologically by fibroblastic activation and increased fibrillogenic activity endothelial and intimal hyperplasia of the arterioles cause vascular occlusion and reduced perfusion [8]. The fibroblastic soft tissue thickening and lack of true soft tissue edema in POPP, as characterized by MRI, is an important feature that differentiates it from osteomyelitis where the edematous nature of the soft tissue swelling is expected to show high signal on $\mathrm{T} 2-\mathrm{W}$ and fluid-sensitive sequences. Furthermore, osteomyelitis might also be expected to show articular and adjacent bone involvement, a feature that is not associated with POPP.

POPP is a rare manifestation of psoriatic arthropathy. Although previously the diagnosis was made primarily on clinical grounds, recent advances in applications of MRI have identified key imaging findings that can help diagnose the condition with greater accuracy and exclude close mimickers, particularly osteomyelitis. MRI also provides the added advantage of picking up early disease activity. With new biologic therapy that has revolutionized the treatment of most systemic arthropathies, it is important that the condition is recognized early on, in order to commence required therapy [9]. 


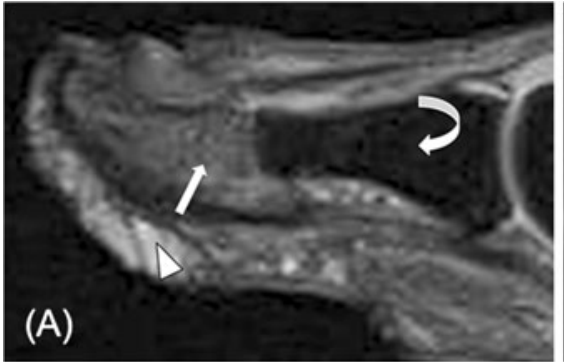

Fig. 4 MRI sagittal (a) and axial proton density (b), once again demonstrating bone marrow edema (arrow) and non-edematous soft tissue thickening (stars) confined to the distal phalanx, and normal marrow within the proximal phalanx (curved arrow). The high signal

Acknowledgments \& Disclosures Dr. Andrew Grainger, Prof. Dennis McGonagle, and Dr. Zoe Ash receive funding from the National Institute of Health Research, Leeds Musculoskeletal Biomedical Research Unit. Jill Halstead receives funding from Arthritis Research, UK.

\section{References}

1. Wollina U, Unger L, Heinig B, Kittner T. Psoriatic arthritis. Dermatol Ther. 2010;23:123-36.

2. Wollina U, Barta U. Arthritis psoriatica - about the spectrum of cutaneous and joint manifestations. Akt Rheumatol. 2000;25:108-12.

3. Reich K, Krüger K, Mössner R, Augustin M. Epidemiology and clinical pattern of psoriatic arthritis in Germany: a prospective interdisciplinary epidemiological study of 1511 patients with plaque-type psoriasis. Br J Dermatol. 2009;160:1040-7.

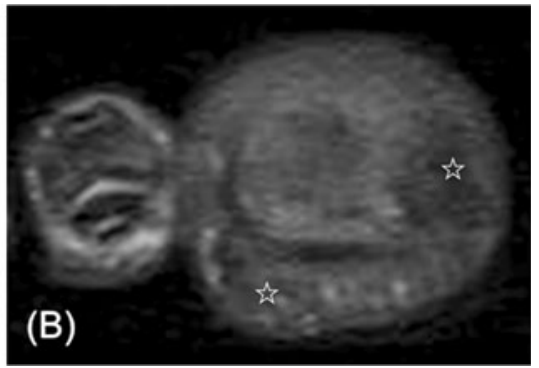

seen on the sagittal image along the plantar aspect of the distal phalanx (triangle) is due to poor peripheral fat suppression and should not be confused with soft tissue edema

4. Fournie B, Viraben R, Durroux R, et al. Psoriatic onycho-pachydermoperiostitis of the big toe. Anatomo-clinical study and physiopatho-genic approach apropos of 4 cases. [French]. Rev Rhum Mal Osteoartic. 1989;56:579-82.

5. Kapusta MA, Dumont C. Differential response of psoriatic onychopachydermo-periostitis to 2 anti-tumor necrosis factor-alpha agents. $\mathrm{J}$ Rheumatol. 2008;35(10):2077-80.

6. Boisseau-Garsaud AM, Beylot-Barry M, Doutre MS, et al. Psoriatic onycho-pachydermo-periostitis. A variant of psoriatic distal interphalangeal arthritis? Arch Dermatol. 1996;132:176-80.

7. Chan C-C, Tsai T-F. Psoriatic onycho-pachydermo-periostitis. Dermatol Sin. 2005;23:86-90.

8. Mattuci-Cerinic M, Ceruso M, Lotti T, et al. The spectrum of dermatological symptoms of pachydermoperiostosis (primary hypertrophic osteoarthropathy). A genetic, cytogenetic and ultrastructural study. Clin Exp Rheumatol. 1992;10 suppl 7:45-8.

9. Bongartz T, Härle P, Friedrich S, Karrer S, Vogt T, Seitz A, et al. Successful treatment of psoriatic onycho-pachydermo periostitis (POPP) with adalimumab. Arthritis Rheum. 2005;52(1):280-2. 